\title{
Effect of TLR4/MyD88 signaling pathway on sepsis-associated acute respiratory distress syndrome in rats, via regulation of macrophage activation and inflammatory response
}

\author{
SHUJUN ZHOU ${ }^{1 *}$, GUI WANG ${ }^{1 *}$ and WENBIN ZHANG ${ }^{2}$ \\ ${ }^{1}$ Department of Critical Care Medicine; ${ }^{2}$ Emergency Department, The Third Affiliated Hospital of Soochow University, \\ The First People's Hospital of Changzhou, Changzhou, Jiangsu 213003, P.R. China
}

Received September 13, 2017; Accepted January 8, 2018

DOI: $10.3892 / \mathrm{etm} .2018 .5815$

\begin{abstract}
The present study aimed to investigate the effects of the Toll-like receptor (TLR)4/myeloid differentiation primary response (MyD)88 signaling pathway on sepsis-associated acute respiratory distress syndrome (ARDS) in rats, and the involvement of macrophage activation and the inflammatory response. A total of 36 specific pathogen-free male Sprague-Dawley rats were selected to establish the rat model of sepsis-associated ARDS using cecal ligation and puncture (CLP). Rats were assigned into the Ab (anti-TLR4 monoclonal antibody)-CLP, CLP and Sham groups. Arterial partial pressure of oxygen $\left(\mathrm{P}_{\mathrm{a}} \mathrm{O}_{2}\right)$ was detected using blood gas analysis. Bronchoalveolar lavage fluid (BALF) and alveolar macrophages were collected. The pathological structure of lung tissue was observed following hematoxylin-eosin staining. The ultrastructural alterations of alveolar epithelial cells were observed under transmission electron microscope. The ratios of wet/dry weight of lung tissue and total protein content in BALF were measured. The concentration of tumor necrosis factor (TNF)- $\alpha$ and interleukin (IL)-1 $\beta$ in BALF and peripheral blood was determined by enzyme-linked immunosorbent assay. The TLR4, TLR9, MyD88 and nuclear factor (NF)- $\mathrm{B}$ mRNA and protein expression levels in alveolar macrophages were measured by reverse transcription-quantitative polymerase chain reaction and western blotting. Compared with the Sham group, the rats in the CLP group demonstrated significantly increased respiratory frequency, lung permeability,
\end{abstract}

Correspondence to: Dr Wenbin Zhang, Emergency Department, The Third Affiliated Hospital of Soochow University, The First People's Hospital of Changzhou, 185 Juqian Street, Changzhou, Jiangsu 213003, P.R. China

E-mail: wenbinzhang953@163.com

*Contributed equally

Key words: TLR4/MyD88 signaling pathway, sepsis, acute respiratory distress syndrome, macrophage activation, inflammatory response lung edema, inflammatory infiltration, TNF- $\alpha$ and IL- $1 \beta$ expression levels in BALF and peripheral blood and TLR4, TLR9, MyD88 and NF- $\mathrm{kB}$ expression levels in macrophages, however decreased arterial $\mathrm{P}_{\mathrm{a}} \mathrm{O}_{2}$. Following pretreatment with anti-TLR4 monoclonal antibody, rats exhibited decreased lung injury, inflammatory infiltration, lung edema, TNF- $\alpha$ and IL-1 $\beta$ expressions in BALF and peripheral blood, and TLR4, TLR9, MyD88 and NF- $\mathrm{kB}$ expression levels in macrophages, with increased arterial $\mathrm{P}_{\mathrm{a}} \mathrm{O}_{2}$. These results suggested that the inhibition of TLR4/MyD88 signaling pathway may relieve sepsis-associated ARDS in rats through regulating macrophage activation and the inflammatory response.

\section{Introduction}

Acute respiratory distress syndrome (ARDS) is reported to be a kind of disease that is characterized by diffuse inflammatory injury of lung and capillary destruction of alveolar, which resulted in alveolar extravasation and acute hypoxemic respiratory failure $(1,2)$. Assorted and numerous factors were reported to be predisposing factors for ARDS, such as drug overdose or abuse, pneumonia, lung contusion, multiple blood transfusions, aspiration of stomach contents and sepsis (3). It has been demonstrated that advanced strategies and pharmacological interventions have been used to patients with ARDS, such as noninvasive positive-pressure ventilation, neuromuscular blockade, $\beta$-adrenergic agonists etc. $(4,5)$. Obviously, in the past 20 years, our awareness of the risk factors and mechanisms of ARDS has been improved, but sepsis-associated ARDS is still related to high mortality $(6,7)$. Several factors, including transforming growth factor- $\beta 1$ (TGF- $\beta$ ) and vascular endothelial (VE) cadherin, were considered to be critical in the pathogenesis of sepsis-associated ARDS $(8,9)$. Hence, it is important to explore molecular mechanism for more effective treatment for patients with sepsis-associated ARDS.

Toll-like receptors (TLRs) are dominating congenital immune receptors to recognize pathogen-associated molecular patterns (PAMPs) that not only initiate the primary response to the invading pathogens but also induce the adaptive immune response (10). Toll-like receptor 4 (TLR4) is a pattern recognition receptor (PPR) that induces inflammatory responses, especially crucial to the progression of antigen-specific 
adaptive immune response, following the identification of some endogenous ligands related to tissue damage (11). Myeloid differentiation primary-response protein 88 (MyD88) act as a shared adaptor molecule for most TLRs which can elicit the production of multiple inflammatory cytokine genes and the activation of nuclear factor $-\kappa \mathrm{B}(\mathrm{NF}-\kappa \mathrm{B})$ and mitogen-activated protein (MAP) kinases involved in neurotoxicity $(12,13)$. Castoldi et al provided evidence that the TLR4/MyD88 signaling pathway was crucial to acute kidney injury (AKI) induced by sepsis (14). The study of Huang et al exerted efforts to identify the role of monoclonal antibody against TLR4 in ventilator-induced lung injury in rats through MyD88/NF- $\kappa$ B signaling (15), which provides some clues for further studies in ARDS. Additionally, TLR4 is one of the key receptors associated with entire body and low-level chronic inflammatory diseases, leading to the macrophage infiltration in diabetic liver injury, through activating $\mathrm{NF}-\kappa \mathrm{B}$ and regulating pro-inflammatory genes (16). Macrophages are heterogeneous cells of innate immune system, playing a critical role in the initiation and resolution of inflammatory response (17). Interestingly, multiple TLRs, especially, TLR4, are involved in macrophage activation and secretion of tumor necrosis factor- $\alpha$ (TNF- $\alpha$ ) (18). From all that mentioned above, it is speculated that TLR4/MyD88 signaling pathway may be related to the activation of macrophages and inflammatory factors, and provide a new therapy for sepsis-associated ARDS. On this regard, this study aims to investigate the role of TLR4/MyD88 signaling pathway in sepsis-associated ARDS via regulating macrophage activation and inflammatory response.

\section{Materials and methods}

Ethics statement. All animal experiments were approved by Ethics Committee of The First People's Hospital of Changzhou, and conducted in accordance with Declaration of Helsinki.

Study subjects. A total of 36 specific pathogen free (SPF) male Sprague-Dawlay (SD) rats (weighted 230 $20 \mathrm{~g}$ ) were purchased from Hunan Slack King Laboratory Animal Co., Ltd. (Changsha, Hunan, China). All rats were housed in separate cages at room temperature of $18-28^{\circ} \mathrm{C}$, with relative humidity of $40-70 \%$, and a 12-h day/night cycle. Rats were fed a standard diet containing $21 \%$ protein and with free access to water. The experiment was carried out after 2 weeks of feeding.

A rat model of sepsis-associated ARDS. Rats were intraperitoneally injected with $40 \mathrm{mg} / \mathrm{kg}$ phenobarbital sodium, and they were fixed on the operating table in the supine position after anesthesia. The abdomen was opened to find the cecum under aseptic condition and the cecum was ligated tightly with a 4 silk thread at the location of $1 / 4$ proximal cecum. A $20 \mathrm{G}$ sterile needle was used to puncture for 3 times in the ligated cecum so as to squeeze a small amount of feces, but fecal pollution should be avoided. The intestinal canal was soaked with $2 \mathrm{ml}$ of $0.9 \%$ sodium chloride solution. The cecum was put back into the abdominal cavity according to its physiological position, and the abdominal cavity was closed layer by layer. After surgery, abdominal subcutaneous injection of
$0.9 \%$ sodium chloride solution $(50 \mathrm{mg} / \mathrm{kg})$ was performed immediately for antishock. In the Sham group, the abdomen of rats was opened and closed without cecal ligation and puncture (CLP). No abdominal subcutaneous injection of $0.9 \%$ sodium chloride solution was performed after surgery (19). After the CLP, TKR-200C small animal ventilator and blood gas analyzer (Jiangxi Teli Anesthesia Breathing Equipment Co., Ltd., Nanchang, Jiangxi, China) were employed to monitor the respiratory frequency and arterial partial pressure of oxygen $\left(\mathrm{P}_{\mathrm{a}} \mathrm{O}_{2}\right)$ of rats.

Animal grouping and model establishment. A total of 36 SD rats after 2-week feeding were randomly divided into 3 groups with 12 rats in each group. The Ab (anti-TLR4 monoclonal antibody)-CLP group: TLR4 antibody $(10 \mathrm{mg} / \mathrm{ml}$; Cell Signaling Technologies, Inc., Beverly, MA, USA) was injected into the caudal vein at 1 day before model establishment, and then the model establishment of sepsis-associated ARDS was conducted. The CLP group: The same volume of normal saline was injected into the caudal vein at 1 day before model establishment, and then the model establishment of sepsis-associated ARDS was conducted. The Sham group: The same volume of normal saline was injected into the caudal vein at 1 day before model establishment, and then the abdomen of rats were opened and closed after anesthesia without CLP.

Arterial blood gas analysis and peripheral blood collection. At $6 \mathrm{~h}$ after the models were established successfully, rats were anaesthetized with intraperitoneal injection of 3\% mebumalnatrium (Sigma-Aldrich; Merck KGaA, Darmstadt, Germany) (30 mg/kg). After successful anesthesia, the rats in the three groups were fully extended and fixed on the operating table in the supine position. Hair of neck, chest and abdomen was shaved using disposable skin preparation knife or disinfection scissors, followed by disinfection. The abdominal cavity was opened with midline abdominal incision to expose the abdominal aorta. Arterial blood $(0.3 \mathrm{ml})$ was collected using $1 \mathrm{ml}$ disposable syringe which had been washed with heparin. Blood gas analysis of blood sample was carried out using blood gas analyzer (Radiometer Medical A/S, Copenhagen, Denmark). The chest was opened to expose the left atrium. The sterile forcep was used to find the right atrium and blood was extracted from the right atrium using a $10 \mathrm{ml}$ sterile syringe. The collected blood was centrifuged at low temperature, and then the supernatant was collected and stored at $-80^{\circ} \mathrm{C}$ for further use.

Bronchoalveolar lavage fluid (BALF) collection. The chest was opened immediately along the anterior midline and the hilum of right lung was ligated with a $2 \mathrm{~mm}$ transverse incision in the trachea. The scalp acupuncture ( $2 \mathrm{~mm}$ outer diameter) removing the pinpoint was applied as tracheal catheter and inserted into the left main bronchus. And then the rats were slowly injected with normal saline $(2.5 \mathrm{ml})$ through the catheter. When the left lung of rats was gradually swelled and the color became pale, the normal saline was slowly pumped back. The lavage step was repeated 5 times with a lavage recovery criterion of the recovery liquid $>2 \mathrm{ml}$ each time. BALF was collected in the $20 \mathrm{ml}$ plastic centrifuge tube with ice bath at 
$4^{\circ} \mathrm{C}$, and then centrifuged in low speed centrifuge at a speed of 4,000 x $\mathrm{g}$ for $10 \mathrm{~min}$. The supernatant was sub-packed and stored at $-80^{\circ} \mathrm{C}$ for the detection of inflammatory cytokines using enzyme-linked immunosorbent assay (ELISA). After centrifugation, the cell sedimentation was resuspended with a pre-cooled RPMI-1640 medium for alveolar macrophage extraction and follow-up experiments.

Alveolar macrophage separation. The BALF collected from the Sham, CLP and Ab-CLP groups was centrifugated, the cell sedimentation of which was added with DMEM medium containing $15 \%$ fetal bovine serum (FBS) in order to resuspend the cells. Cell suspension was placed in the 6 -well plates with adherent culture for $2 \mathrm{~h}$ under $5 \% \mathrm{CO}_{2}$ at $37^{\circ} \mathrm{C}$. After removal of the cell culture medium, the adherent alveolar macrophages were collected. After wright staining, cells with the gray cytoplasm and the purple red nucleus under microscopic examination were confirmed as macrophage with a purity of $>95 \%$. Cells were observed under the microscope after trypan blue exclusion test. The dead cells were stained as light blue, while the living cells were not stained. Cell viability was calculated in accordance with the following formula: Cell viability $(\%)=($ the number of total cells-the number of stained cells)/the number of total cells x $100 \%$. From the above formula, cell viability (cell viability of normal cells $>95 \%$ ) was calculated, which indicated that the cells could be used for subsequent experiments with good growth and strong viability (20).

Lung tissue extraction. After lavage of the left lung alveolar, the upper lobe of the right lung was immediately resected and fixed in formaldehyde solution for pathological examination. After the middle lobe of the right lung was resected, the surface of the lobe was rinsed immediately with normal saline and dried with a filter paper, and then it was weighed as wet weight. After that, it was put in a $65^{\circ} \mathrm{C}$ oven and baked for at least $48 \mathrm{~h}$. Then, it was weighed repeatedly to obtain constant dry weight. The ratios of wet weight/dry weight (W/D) were calculated. After the lower lobe of the right lung was resected, the blood in the surface was washed using the $\mathrm{ddH}_{2} \mathrm{O}$ of RNA-Free and DNA-Free. The lower lobe of the right lung was cut into two parts, and sub-packed with tinfoil which was baked in a $260^{\circ} \mathrm{C}$ oven in order to inactivate enzyme. After that it was quickly stored in liquid nitrogen. Then, it was transferred and stored in refrigerator at $-80^{\circ} \mathrm{C}$. One part was used for quantitative polymerase chain reaction (qPCR) and the other for western blotting.

Hematoxylin and eosin $(H \& E)$ staining. The samples were embedded in paraffin and sliced into small sections of $5 \mu \mathrm{m}$ thickness. The sections were routinely dewaxed with xylene for $30 \mathrm{~min}$, followed by gradient ethanol dehydration with $100 \%$ ethanol, $95 \%$ ethanol, $85 \%$ ethanol and $70 \%$ ethanol, respectively for $2 \mathrm{~min}$. The tissue sections were immersed in hematoxylin for $15 \mathrm{~min}$. Then the tissue sections were immersed in $1 \%$ hydrochloric alcohol for 6-8 sec and in saturated lithium carbonate solution for $1 \mathrm{~min}$. Subsequently, the tissue sections were immersed in eosin staining solution for $4 \mathrm{~min}(21)$.

Transmission electron microscope (TEM) observation. The lung tissue $(1.0 \times 1.0 \times 1.0 \mathrm{~mm})$ was washed by phosphate buffer saline (PBS) pre-cooled at $4^{\circ} \mathrm{C}$, and then the tissue was fixed with $4 \mathrm{ml}$ of $2 \%$ glutaraldehyde (PH 7.4; pre-cooled at $4^{\circ} \mathrm{C}$ ) for $30 \mathrm{~min}$. The lung tissue was rinsed by PBS for 3 times, 10 min each time. The lung tissue was fixed in $1 \%$ osmium acid at $4^{\circ} \mathrm{C}$ for $30 \mathrm{~min}$, following which the lung tissue was rinsed by PBS for 3 times. The lung tissue was dehydrated at room temperature using gradient concentrations of acetone: $50 \%$ acetone for once with 10 min each time, $70 \%$ acetone for once with 10 min each time, $90 \%$ acetone for twice with 10 min each time and $100 \%$ acetone for three times with $10 \mathrm{~min}$ each time. After the dehydrating agent in the bottle was discarded, the lung tissue was embedded with $3 \mathrm{ml}$ of pure acetone-EPON812 for $30 \mathrm{~min}$ at room temperature. After the removal of the diluted embedding agent, the lung tissue was embedded with $1 \mathrm{ml}$ of pure embedding agent overnight at room temperature. Two drops of the mixed embedding medium were dropped to the bottom of the capsule module hole. After moving the lung mass to the center of the module, it was filled with the mixed embedding medium and then baked in a $60^{\circ} \mathrm{C}$ oven for $24 \mathrm{~h}$. The embedding block was trimmed for the preparation of semi-thin sections, and ultrathin sections were prepared after accurate positioning. The sections were stained using lead acetate uranium, after which the tissue sections were observed under TEM (Hitachi High-Technologies Corporation, Tokyo, Japan).

Measurement of lung wet weight/dry weight (W/D) ratios. Part of tissues in the middle lobe of the right lung was selected and the exudate and blood on the surface of which was absorbed using filter paper. The wet weight of the lung was weighed using the electronic balance (Mettler-Toledo International Inc., Zurich, Switzerland) and then baked in a $70^{\circ} \mathrm{C}$ electrothermal constant-temperature dry box (Shanghai Jinghong Laboratory Instrument Co., Ltd., Shanghai, China) until it weighed to constant weight as dry weight. The lung coefficient was calculated in accordance with the following formula: $W / D=$ wet weight of lung/dry weight of lung, lung water content=(net wet weight of lung-net dry weight of lung)/net wet weight of lung x $100 \%$.

Enzyme-linked immunosorbent assay (ELISA). The BALF and peripheral blood that collected and stored separately in the refrigerator was defrosted on the ice. The ELISA kit was taken out from the $4^{\circ} \mathrm{C}$ refrigerator, and maintained at room temperature for $30 \mathrm{~min}$. The ELISA kit for TNF- $\alpha$ and interleukin-1 $\beta$ (IL-1 $\beta$ ) was purchased from Cusabio Biotech Co., Ltd. (Wuhan, China), and the experimental procedures were carried out in strict accordance with the instructions of kit. The blank well was applied for zero calibration and the optical density (OD) value of each well was measured successively using a microplate reader (Bio-Tek Instruments Inc., Winooski, Vermont, USA) at a wavelength of $450 \mathrm{~nm}$.

Reverse transcription- $q P C R(R T-q P C R)$. The TLR4, TLR9, MyD88 and NF- $\kappa \mathrm{B}$ mRNA expressions in alveolar macrophage were measured by qRT-PCR. The total RNA was extracted from alveolar macrophage using trizol method (Takara Biotechnology Co., Ltd., Dalian, Liaoning, China), and the concentration 
and purity of RNA were detected. Reverse transcription kit (DRR047S; Takara Biotechnology Co., Ltd.) was used to reversely transcribe RNA into cDNA with $10 \mu \mathrm{l}$ of reverse transcription system. The cDNA obtained from above reversion was diluted with $65 \mu \mathrm{l}$ of diethyl pyrocarbonate (DEPC) solution with sufficient mixing. Reaction system: $5 \mu 1$ of SsoFast EvaGreen Supermix (1708882; Bio-Rad Laboratories, Inc., Hercules, CA, USA), $0.5 \mu \mathrm{l}$ of Forward primer $(10 \mu \mathrm{M}), 0.5 \mu \mathrm{l}$ of Reverse primer $(10 \mu \mathrm{M})$ and $4 \mu \mathrm{l}$ of cDNA. PCR amplification conditions: Pre-denaturation at $95^{\circ} \mathrm{C}$ for $1 \mathrm{~min}, 30$ cycles of denaturation at $95^{\circ} \mathrm{C}$ for $30 \mathrm{sec}$ and annealing at $58^{\circ} \mathrm{C}$ for $5 \mathrm{sec}$, and final extension at $72^{\circ} \mathrm{C}$ for $5 \mathrm{sec}$. Primers were synthesized by Beijing Genomics Institute (BGI, Shenzhen, China) (Table I). $\beta$-actin was used as an internal reference. Each gene in the samples was measured in triplicates. The reliability of the PCR results was verified using the solubility curve. The $\mathrm{C}_{\text {quantification }}$ cycle $(\mathrm{Cq})$ value was obtained. Formula: $\Delta \mathrm{Cq}=\mathrm{Cq}_{\text {target gene }}-\mathrm{Cq}_{\text {in- }}$ ternal reference, $\Delta \Delta \mathrm{Cq}=\Delta \mathrm{Cq}_{\text {the experimental group }}-\Delta \mathrm{Cq}_{\text {the control group. }}$. And the gene expressions were calculated using $2^{-\Delta \Delta \mathrm{Cq}}$ method (22). The experiment was repeated three times.

Western blotting. Alveolar macrophages were collected and digested with trypsin. After centrifugation, alveolar macrophages were washed by PBS for two times, and then cracked with pre-cooled cell lysate on ice for $30 \mathrm{~min}$. Then, they were centrifuged at $12,000 \mathrm{x} \mathrm{g}$ for $20 \mathrm{~min}$ at $4^{\circ} \mathrm{C}$, after which the supernatant was collected and stored at $-20^{\circ} \mathrm{C}$. The $2 \mu \mathrm{g} / \mu \mathrm{l}$ of bovine serum albumin (BSA) was diluted with PBS into following concentrations: $20,15,10,5,2.5,0 \mu \mathrm{g} / \mathrm{ml}$. bicinchoninic acid (BCA) protein assay kit (Thermo Fisher Scientific, Inc., Waltham, MA, USA) was used to detect protein concentration in accordance with the procedure of instructions combined with sample number. Protein electrophoresis was performed in a $4^{\circ} \mathrm{C}$ chromatography cabinet. The electrophoresis was performed with compressed gels at the voltages of $80 \mathrm{~V}$ and then separation gels at $120 \mathrm{~V}$. After electrophoresis, the proteins were transferred onto polyvinylidene fluoride (PVDF) membrane using wet transfer, and then they were blocked using 5\% skimmed milk-tris-buffered saline (TBST) and incubated at room temperature for $2 \mathrm{~h}$. Protein samples were incubated at $4^{\circ} \mathrm{C}$ for overnight after being added with primary antibodies against TLR4 (1:500; Cell Signaling Technologies, Inc.), TLR9 (1:800), MyD88 (1:900), NF-кB $(1: 1,000)$, and GAPDH $(1: 8,000$; Proteintech Group, Inc. Chicago, IL, USA). Samples were rinsed by TBST three times, 10 min each time. Samples were added with the second antibody (1:10,000), and incubated at room temperature for $1 \mathrm{~h}$. Then, samples were rinsed by TBST three times, $10 \mathrm{~min}$ each time. Finally, the samples were stained using chemiluminescence and tabulated by X-ray, followed by film development and fixation, and finally results were analyzed.

Statistical analysis. Data were analyzed statistically using SPSS 22.0 software (IBM Corp, Armonk, NY, USA). Measurement data were presented as the mean \pm standard deviation (SD). The comparisons among multiple groups were conducted by the one-way analysis of variance (ANOVA), and the comparisons between two groups were tested by the t-tests. $\mathrm{P}<0.05$ was considered to indicate a statistically significant difference.
Table I. Primer sequences for quantitative polymerase chain reaction.

\begin{tabular}{ll}
\hline Gene & \multicolumn{1}{c}{ Primer sequences $\left(5^{\prime}-3^{\prime}\right)$} \\
\hline TLR4 & \\
F & CGCTCTGGCATCATCTTCAT \\
R & CTCCTCAGGTCAAAGTTGTTGC \\
TLR9 & \\
F & CCTGGCACACAATGACATTCA \\
R & TAAAGGTCCTCCTCGTCCCA \\
MyD88 & \\
F & GAGATCCGCGAGTTTGAGAC \\
R & TTGTCTGTGGGACACTGCTC \\
NF- $\kappa$ B & \\
F & \\
R & GAGGACTTGCTGAGGTTGG \\
GAPDH & TGGGGTGGTTGATAAGGAGTG \\
F & \\
R & AACGGATTTGGTCGTATTGGG- \\
\hline
\end{tabular}

F, forward; R, reverse; TLR, Toll-like receptor; MyD88, myeloid differentiation primary response 88 ; NF- $\kappa \mathrm{B}$, nuclear factor- $\mathrm{\kappa B}$.

\section{Results}

Respiratory frequency and arterial $\mathrm{P}_{a} \mathrm{O}_{2}$ of rats in three groups. The respiratory frequencies of rats in the Sham, CLP and Ab-CLP groups were $78 \pm 5,121 \pm 7$ and $86 \pm 8$ breaths/min, respectively. The respiratory frequencies of rats in the CLP group were significantly higher than that in the Sham and Ab-CLP groups $(\mathrm{P}<0.05)$. Arterial $\mathrm{P}_{\mathrm{a}} \mathrm{O}_{2}$ in the Sham, CLP and Ab-CLP groups were 79.3 $\pm 8.9,54.6 \pm 7.2$ and $74.9 \pm 6.9 \mathrm{mmHg}$, respectively. In comparison to the CLP group, the Sham and $\mathrm{Ab}-\mathrm{CLP}$ groups showed decreased arterial $\mathrm{P}_{\mathrm{a}} \mathrm{O}_{2}(\mathrm{P}<0.05)$ (Fig. 1).

Pathological changes of lung tissue of rats in three groups. Lung tissue samples were observed under the light microscope. In the Sham group, the lung tissue structure was complete and clear, and less interstitial inflammatory cell infiltration was visible in alveolar without obvious congestion and hemorrhage. Besides, the alveolar septum in the Sham group was not widened, and the alveolar wall was complete without edema in the cavity. In the CLP group, a large area of alveolar wall was destructed in the lung tissue of rats. Additionally, the alveolar cavity was filled with pink edema fluid with widened alveolar septum and edema. A large number of inflammatory cells were infiltrated and red blood cells (RBCs) were leaked out with pulmonary vascular congestion. In the Ab-CLP group, the lung tissue of rats still had the same pathological changes as that in the CLP group, but the size of damaged area, alveolar edema fluid, alveolar interstitial edema, inflammatory cell infiltration and leakage of RBCs were significantly reduced (Fig. 2).

Cellular structure of type II alveolar epithelial cells under transmmision electron microscope. The results of high 
A

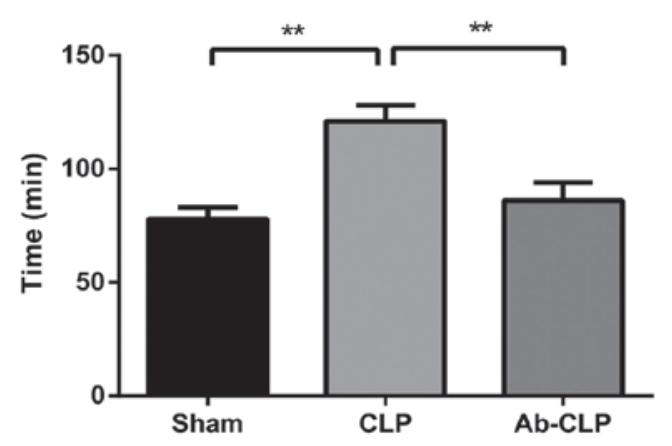

B

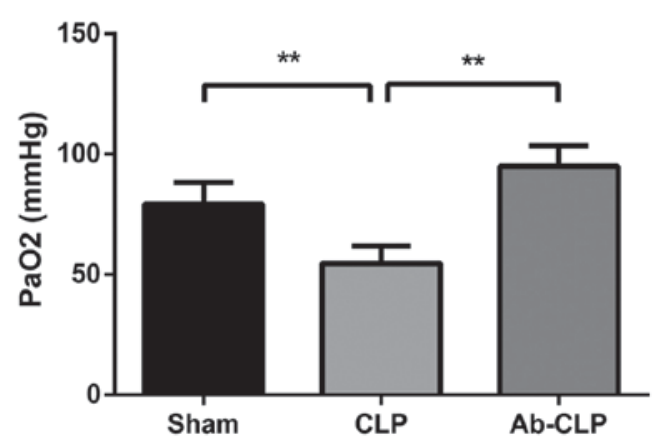

Figure 1. Respiratory frequency and arterial $\mathrm{P}_{\mathrm{a}} \mathrm{O}_{2}$ of rats in three groups. (A) Comparison of respiratory frequency of rats among the Sham, CLP and $\mathrm{Ab}-\mathrm{CLP}$ groups; (B) comparison of the arterial $\mathrm{P}_{\mathrm{a}} \mathrm{O}_{2}$ of rats among the Sham, CLP and Ab-CLP groups by blood gas analysis; ${ }^{* *} \mathrm{P}<0.005$ compared with the CLP group. $\mathrm{P}_{\mathrm{a}} \mathrm{O}_{2}$, partial pressure of oxygen; CLP, cecal ligation puncture; Ab, anti-TLR4 monoclonal antibody.
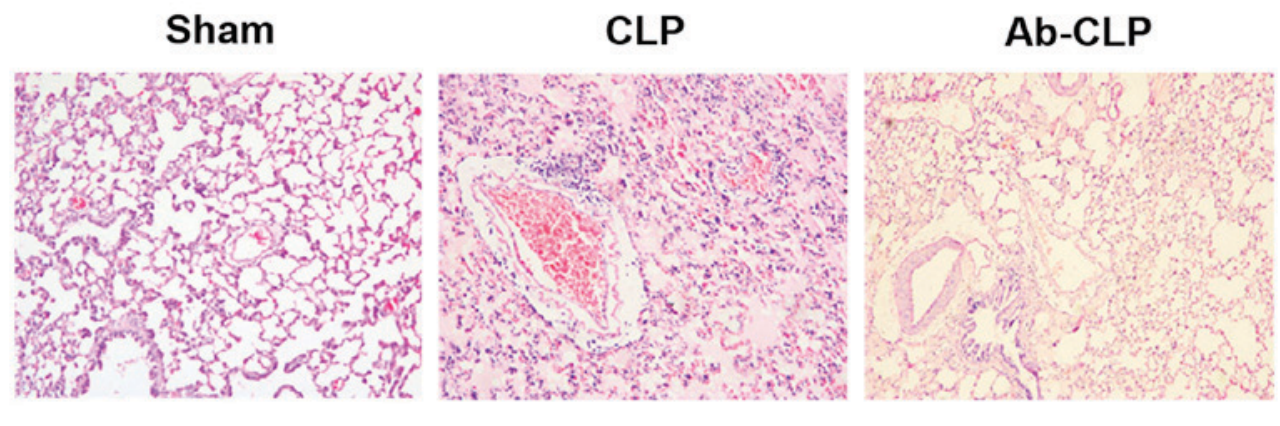

Figure 2. Pathological structure of lung tissue of rats by H\&E staining in three groups (magnification, $\mathrm{x} 200$ ). H\&E, hematoxylin and eosin; CLP, cecal ligation puncture; Ab, anti-TLR4 monoclonal antibody.
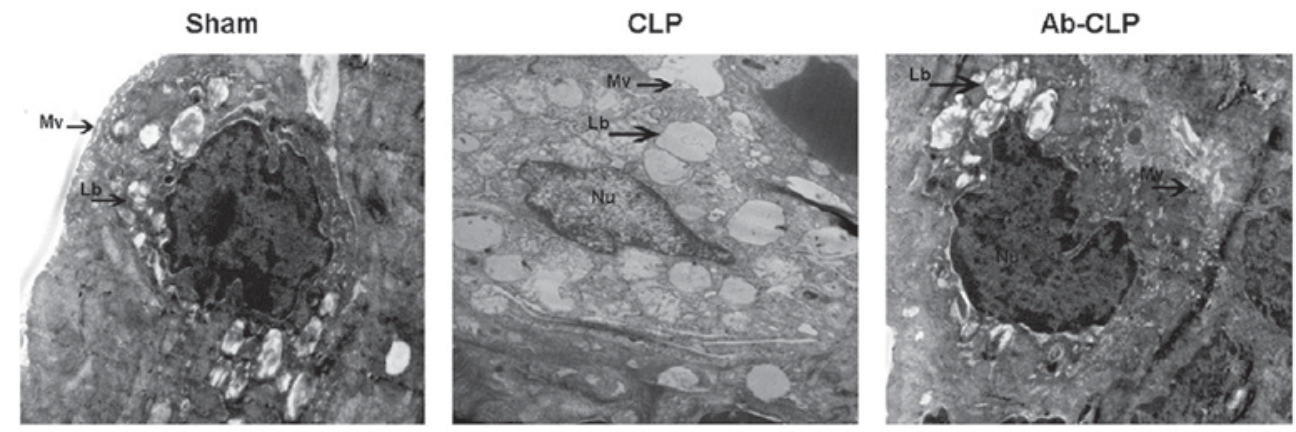

Figure 3. Cellular structure of type II alveolar epithelial cells of rats in three groups under transmmision electron microscope (magnification, x2,800). CLP, cecal ligation puncture; Ab, anti-TLR4 monoclonal antibody; Lb, Lamellar bodies; Mv, Microvilli; Nu, Nucleus.

resolution transmission electron microscope are shown in Fig. 3. In the CLP group, the nucleus and its boundary was clear with karyopyknosis and obvious shape changing; the nucleolus and the cell membrane were broken with disordered cytoplasm structure and a large number of vacuoles; the microvillus almost disappeared with badly damaged organelle, vague shape of nucleus and obvious perinuclear space; the nucleolus presented apoptosis status with obvious exudation in alveolar space. The type II alveolar epithelial cells have integrated cell membrane in the Sham and Ab-CLP groups. Meanwhile, the cytoplasm and the nuclear structure were clear with a small amount of cytoplasmic cavitation and slight perinuclear space but no obvious cell damage in the Sham and Ab-CLP groups. The chromatin is evenly aligned with continuous and integrated cell membrane.

Comparisons of lung W/D ratios and total protein content in $B A L F$ of rats in three groups. In the Sham, CLP and Ab-CLP groups, lung W/D ratios were $4.214 \pm 0.324 ; 6.678 \pm 0.241$ and $5.901 \pm 0.212$, respectively. The lung W/D ratios in the CLP group were significantly higher than that in the Sham and Ab-CLP groups $(\mathrm{P}<0.05$; Fig. $4 \mathrm{~A})$. In consistent with the results of pathological examination, it was suggested that the lung tissue of rats in the CLP group had obvious pulmonary edema. The W/D ratios of lung tissue were reduced after 
A

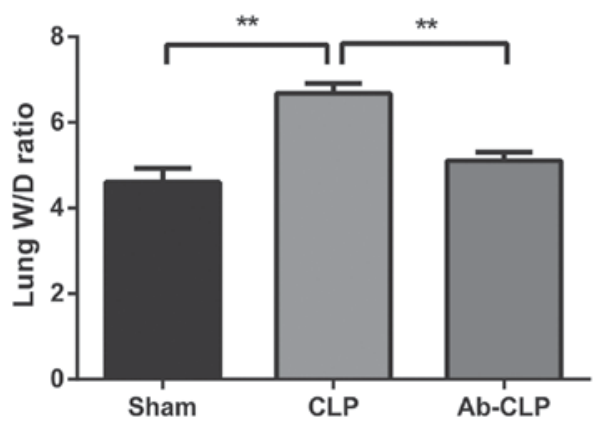

B

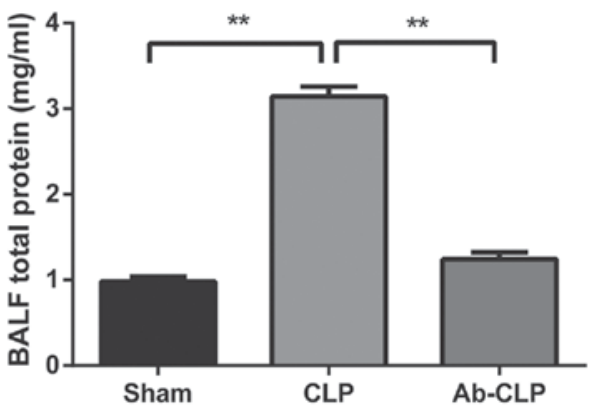

Figure 4. Comparisons of W/D ratios and total protein content in BALF of rats in three groups. (A) Comparison of the W/D ratios of rats among the Sham, CLP and Ab-CLP groups; (B) comparison of the total protein content in BALF of rats among the Sham, CLP and Ab-CLP groups; ${ }^{* *} \mathrm{P}<0.005$ compared with the CLP group. W/D, wet weight/dry weight; BALF, bronchoalveolar lavage fluid; CLP, cecal ligation puncture; Ab, anti-TLR4 monoclonal antibody.

A

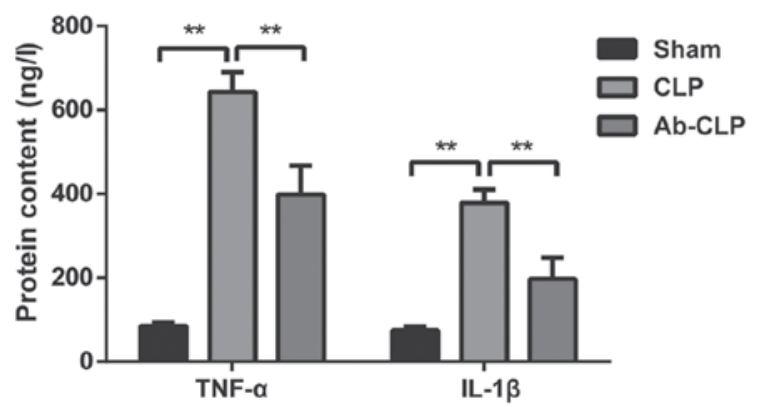

B

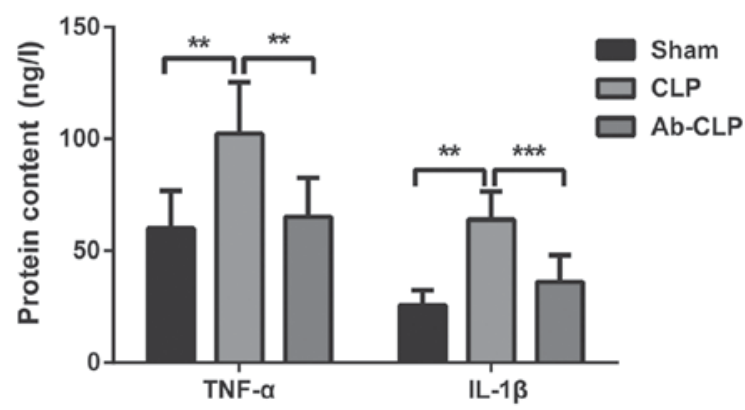

Figure 5. Comparison of the protein contents of inflammatory factors c in BALF and peripheral blood among the three groups. (A) Comparison of the protein contents of inflammatory factors TNF- $\alpha$ and IL-1 $\beta$ in BALF among the Sham, CLP and Ab-CLP groups; (B) comparison of the protein contents of inflammatory factors TNF- $\alpha$ and IL-1 $\beta$ in peripheral blood among the Sham, CLP and Ab-CLP groups; ${ }^{* *} \mathrm{P}<0.005$ compared with the CLP group; ${ }^{* * *} \mathrm{P}<0.001$ compared with the CLP group. BALF, bronchoalveolar lavage fluid; CLP, cecal ligation puncture; Ab, anti-TLR4 monoclonal antibody; TNF- $\alpha$, tumor necrosis factor- $\alpha$; IL-1 $\beta$, interleukin-1 $\beta$.

A
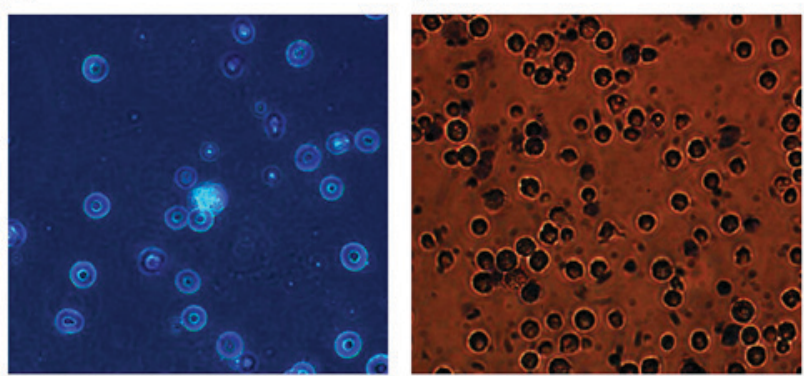

Figure 6. Images of alveolar macrophage after wright-giemsa staining and trypan blue staining (magnification, x400). (A) Image of alveolar macrophage after wright-giemsa staining; (B) image of alveolar macrophage after trypan blue staining.

pretreatment with anti-TLR4 monoclonal antibody (anti-TLR4 $\mathrm{mAb}$ ), which indicated that CLP could reduce the degree of pulmonary edema after pretreatmentm of anti-TLR $4 \mathrm{mAb}$. In comparison to the CLP group, the total protein contents in BALF in the Sham and Ab-CLP groups were evidently decreased $(\mathrm{P}<0.05$; Fig. 4B). It was suggested that the total protein content in BALF was significantly increased in the CLP group, but that was decreased after pretreatment with anti TLR-4 mAb.
Pretreatment with anti TLR $4 \mathrm{mAb}$ reduced the protein contents of inflammatory factors (TNF- $\alpha$ and $I L-1 \beta)$ in BALF and peripheral blood. The protein contents of TNF- $\alpha$ and IL-1 $\beta$ in BALF and peripheral blood of rats in three groups were measured using ELISA (Fig. 5). The results showed that the protein contents of TNF- $\alpha$ and IL- $1 \beta$ in the Sham group were $85.14 \pm 7.82$ and $75.16 \pm 8.62$, respectively. The protein contents of TNF- $\alpha$ and IL-1 $\beta$ in the CLP group were $643.15 \pm 47.18$ and $378.06 \pm 32.19$, respectively. The protein contents of TNF- $\alpha$ and IL-1 $\beta$ in the Ab-CLP group were 398.46 \pm 69.12 and $197.15 \pm 50.37$, respectively. The protein contents of TNF- $\alpha$ and IL-1 $\beta$ in the CLP group were significantly higher than these in the Sham and Ab-CLP groups $(\mathrm{P}<0.05)$. It was suggested that the pretreatment with anti-TLR $4 \mathrm{mAb}$ reduced the synthesis and secretion of inflammatory factors induced by sepsis-associated ARDS, and alleviated the inflammatory response.

The purity and cell viability of alveolar macrophage. Alveolar macrophages were obtained by bronchoalveolar lavage with a purity over $95 \%$. The isolated alveolar macrophages were round or oval with clear and neat edge, the cytoplasm of which was slightly gray with pale purple red cytoplasmic granules and deep purple red nucleus in the cells. The purple-red nucleus and the pale red cytoplasm 
A

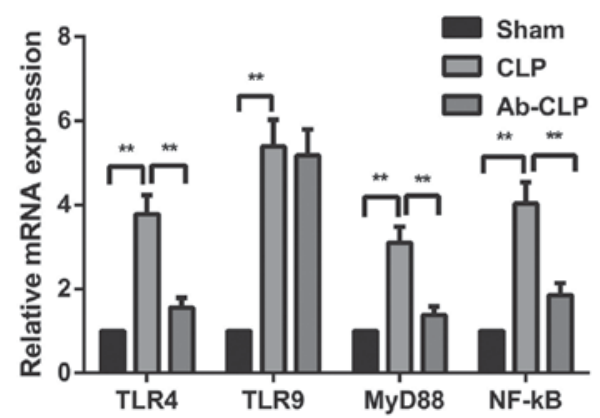

B

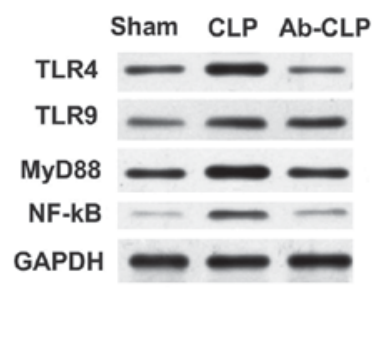

C

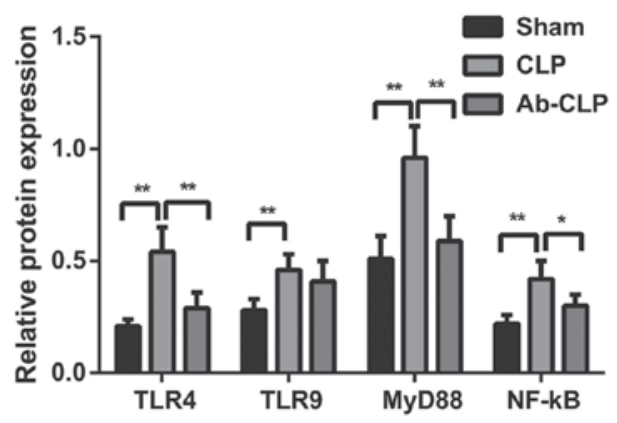

Figure 7. Comparison of the mRNA and protein expressions of TLR4/MyD88 signaling pathway-related factors in alveolar macrophage among the three groups. (A) Comparison of TLR4, TLR9, MyD88 and NF- $\mathrm{B}$ P65 mRNA expressions in alveolar macrophage of rats among the Sham, CLP and Ab-CLP groups; (B) protein bands of TLR4, TLR9, MyD88 and NF- $\mathrm{BB}$ P65 in the Sham, CLP and Ab-CLP detected by western blotting; (C) comparison of TLR4, TLR9, MyD88 and NF-кB P65 protein expressions in alveolar macrophage of rats among the Sham, CLP and Ab-CLP groups; ${ }^{* *}$ P $<0.005$ compared with the CLP group; ${ }^{*} \mathrm{P}<0.05$ compared with the CLP group. CLP, cecal ligation puncture; Ab, anti-TLR-4 monoclonal antibody; TLR4, Toll-like receptor 4; TLR9, Toll-like receptor 9; MyD88, myeloid differentiation primary-response protein 88; NF- $\kappa \mathrm{B}$, nuclear factor- $\kappa \mathrm{B}$; GAPDH, glyceraldehyde-3-phosphate dehydrogenase.

were occasionally visible with pale purple red neutrophile granulocyte. As shown in Fig. 6, cell viability is $>95 \%$ after trypan blue staining.

Pretreatment with anti-TLR4 $\mathrm{mAb}$ inhibited TLR4/MyD88 signaling pathway in alveolar macrophage of rats with sepsis-associated ARDS. The mRNA and protein expressions of TLR4/MyD88 signaling pathway-related factors (TLR4, TLR9, MyD88 and NF-кB P65) in alveolar macrophage of rats with sepsis-associated ARDS were measured by qRT-PCR and western blotting (Fig. 7). The results showed that TLR4/MyD88 signaling pathway was activated after CLP, and the expressions of TLR4, MyD88, $\mathrm{NF}-\kappa \mathrm{B}$ P65 mRNAs and proteins in the CLP group were significantly higher than these in the Sham group $(\mathrm{P}<0.05)$. In comparison to the CLP group, the Ab-CLP group showed the inactivation of TLR4/MyD88 signaling pathway after pretreatment with anti-TLR4 $\mathrm{mAb}$. There was no significant difference in the TLR9 protein and mRNA expressions between the CLP and Ab-CLP groups. But TLR9 protein and mRNA expressions in the CLP and Ab-CLP groups were significantly higher than that in the Sham group $(\mathrm{P}<0.05)$. It was suggested that pretreatment with anti-TLR4 $\mathrm{mAb}$ inhibited the TLR4/MyD88 signaling pathway in alveolar macrophage pf rats with sepsis-associated ARDS.

\section{Discussion}

Over the past few years, TLR4/MyD88 signaling pathway plays a crucial role in various diseases including cerebral ischemia/reperfusion injury, diabetic liver injury and ventilator-induced lung injury $(16,23,24)$. In this study, we further investigated the effects of TLR4/MyD88 signaling pathway on rats with sepsis-associated ARDS by regulating macrophage activation, and found that the inhibition of TLR4/MyD88 signaling pathway may relieve sepsis-associated ARDS through regulating macrophage activation and inflammatory response.

One of the main findings in our study was that in comparison to the CLP group, the Ab-CLP group showed significantly decreased damaged area, alveolar edema fluid, alveolar interstitial edema, inflammatory cell infiltration and leakage of red blood cells (RBCs) which indicated that the pretreatment with anti-TLR4 mAb alleviated lung injury, pulmonary edema and inflammatory infiltration of lung tissue in a rat model of sepsis-associated ARDS. Rats in the Ab-CLP group were treated with anti-TLR4 mAb before CLP. Importantly, $\mathrm{mAbs}$ account for a large proportion of medicines in effective treatments for the patients with a variety of inflammatory diseases, and mAbs are created to inhibit TLR4 signaling pathway with a Fc $\gamma \mathrm{R}$-binding mechanism (25). In line with our study, Huang et al also demonstrated that anti-TLR4 mAb attenuated the lung injury caused by mechanical ventilation, inflammation and edema in rats by inhibiting TLR4/MyD88 signaling pathway (15), but our study indicated that inhibition of TLR4/MyD88 signaling pathway may relieve sepsis-associated ARDS in rats through regulating macrophage activation and inflammatory response. Thus, the inhibition of TLR4/MyD88 signaling pathway might contribute to less lung injury, inflammation and edema in rats with sepsis-associated ARDS.

Additionally, in our study, the results of ELISA indicated the expressions of TNF- $\alpha$ and IL- $1 \beta$ in the CLP group was significantly higher than that in the Sham and Ab-CLP groups, suggesting that pretreatment of anti-TLR4 $\mathrm{mAb}$ reduced the synthesis and secretion of inflammatory factors in sepsis-associated ARDS, and alleviated the inflammatory response. Macrophage responses are coordinated through classical (or M1) and alternative (or M2) activation programs (26). The functions of macrophage are greatly determined by their activation states, exerting a causal role of macrophage activation in the initiation of inflammation (27). TNF- $\alpha$ and IL-1 $\beta$ were major pro-inflammatory cytokines and both were mainly created by the activated macrophages (28). A study conducted by Lin et al once demonstrated that the inhibition of TLR4/MyD88 signaling pathway reduced the expressions of proinflammatory cytokines IL-1 $\beta$ and TNF- $\alpha$, thereby ameliorating the proinflammatory phenotype (29), which is in consistent with our investigation. Inflammatory cytokines, including IL-2, IL-4, IL6, IL8 and IL-1 $\beta$, correlates with the mortality of ARDS $(30,31)$. From the results above, we see that the inhibition of the TLR4/MyD88 
signaling pathway may have protective effects on sepsis-associated ARDS through suppressing inflammatory response, which could be regulated by macrophage activation.

Surprisingly, the results revealed that TLR4/MyD88 signaling pathway was activated after CLP, the mRNA and protein expressions of TLR4, MyD88, NF- $\kappa$ B P65 in the CLP group was significantly higher than that in the Sham group. While in comparison to the CLP group, the Ab-CLP group showed the inactivation of TLR4/MyD88 signaling pathway after pretreatment with anti-TLR4 mAb. TLR4 signaling in macrophages regulates hundreds of gene expressions stimulating anti-microbial activity and induces inflammatory signaling pathways driving inflammatory responses (32). Dai et al revealed that the high expression of TLR4 and TLR9 in alveolar macrophage played a critical role in ventilator-induced lung injury, through the recruitment of MyD88, which could activate NF- $\kappa \mathrm{B}$ inducing the transcription of various pro-inflammatory genes (33). NF- $\kappa \mathrm{B}$ acts as a nuclear factor, the secretion of pro-inflammatory cytokines, such as IL-6, IL-8, and TNF- $\alpha$, that could be mediated by the MyD88 signaling (34). Hence, from all that mentioned above, the pretreatment with anti-TLR $4 \mathrm{mAb}$ inhibited the expressions of TLR4, decreased the secretion of MyD88 thereby suppressing the activation of $\mathrm{NF}-\kappa \mathrm{B}$, which reduced the inflammatory response to sepsis-associated ARDS.

Consequently, our present study provided evidence that the inhibition of TLR4/MyD88 signaling pathway might relieve sepsis-associated ARDS in rats through regulating macrophage activation. It is believed that the pretreatment of anti-TLR4 mAb inhibited the TLR4/MyD88 signaling pathway and alleviated inflammatory response. Further studies are needed to understand the molecular mechanism underlying the role of TLR4/MyD88 signaling pathway in sepsis-associated ARDS and thus to support its potential clinical application.

\section{References}

1. Han S and Mallampalli RK: The acute respiratory distress syndrome: From mechanism to translation. J Immunol 194: 855-860, 2015.

2. Lin WC, Chen CW, Huang YW, Chao L, Chao J, Lin YS and Lin CF: Kallistatin protects against sepsis-related acute lung injury via inhibiting inflammation and apoptosis. Sci Rep 5: $12463,2015$.

3. Wang M, Yan J, He X, Zhong Q, Zhan C and Li S: Candidate genes and pathogenesis investigation for sepsis-related acute respiratory distress syndrome based on gene expression profile. Biol Res 49: 25, 2016.

4. Xu Z, Huang Y, Mao P, Zhang J and Li Y: Sepsis and ARDS: The dark side of histones. Mediators Inflamm 2015: 205054, 2015.

5. Boyle AJ, Mac Sweeney R and McAuley DF: Pharmacological treatments in ARDS; a state-of-the-art update. BMC Med 11: 166, 2013.

6. Dizier S, Forel JM, Ayzac L, Richard JC, Hraiech S, Lehingue S, Loundou A, Roch A, Guerin C and Papazian L; ACURASYS study investigators; PROSEVA Study Group: Early hepatic dysfunction is associated with a worse outcome in patients presenting with acute respiratory distress syndrome: A post-Hoc analysis of the ACURASYS and PROSEVA studies. PLoS One 10: e0144278, 2015.

7. Mansur A, Steinau M, Popov AF, Ghadimi M, Beissbarth T, Bauer $M$ and Hinz J: Impact of statin therapy on mortality in patients with sepsis-associated acute respiratory distress syndrome (ARDS) depends on ARDS severity: A prospective observational cohort study. BMC Med 13: 128, 2015.
8. de Pablo R, Monserrat J, Reyes E, Díaz D, Rodríguez-Zapata M, la Hera Ad, Prieto A and Alvarez-Mon M: Sepsis-induced acute respiratory distress syndrome with fatal outcome is associated to increased serum transforming growth factor beta-1 levels. Eur J Intern Med 23: 358-362, 2012.

9. Herwig MC, Tsokos M, Hermanns MI, Kirkpatrick CJ and Müller AM: Vascular endothelial cadherin expression in lung specimens of patients with sepsis-induced acute respiratory distress syndrome and endothelial cell cultures. Pathobiology 80 : 245-251, 2013.

10. Avlas O, Fallach R, Shainberg A, Porat E and Hochhauser E: Toll-like receptor 4 stimulation initiates an inflammatory response that decreases cardiomyocyte contractility. Antioxid Redox Signal 15: 1895-1909, 2011.

11. Liu L, Gu H, Liu H, Jiao Y, Li K, Zhao Y, An L and Yang J: Protective effect of resveratrol against IL-1 $\beta$-induced inflammatory response on human osteoarthritic chondrocytes partly via the TLR4/MyD88/NF- $\kappa$ B signaling pathway: An 'in vitro study'. Int J Mol Sci 15: 6925-6940, 2014

12. Kim KH, Jo MS, Suh DS, Yoon MS, Shin DH, Lee JH and Choi KU: Expression and significance of the TLR4/MyD88 signaling pathway in ovarian epithelial cancers. World J Surg Oncol 10: 193, 2012.

13. Zhu HT, Bian C, Yuan JC, Chu WH, Xiang X, Chen F, Wang CS, Feng $\mathrm{H}$ and Lin JK: Curcumin attenuates acute inflammatory injury by inhibiting the TLR4/MyD88/NF- $\kappa \mathrm{B}$ signaling pathway in experimental traumatic brain injury. J Neuroinflammation 11: 59, 2014.

14. Castoldi A, Braga TT, Correa-Costa M, Aguiar CF, Bassi ÊJ, Correa-Silva R, Elias RM, Salvador F, Moraes-Vieira PM, Cenedeze MA, et al: TLR2, TLR4 and the MYD88 signaling pathway are crucial for neutrophil migration in acute kidney injury induced by sepsis. PLoS One 7: e37584, 2012.

15. Huang C, Pan L, Lin F, Dai H and Fu R: Monoclonal antibody against Toll-like receptor 4 attenuates ventilator-induced lung injury in rats by inhibiting MyD88- and NF- $\kappa \mathrm{B}$-dependent signaling. Int J Mol Med 39: 693-700, 2017.

16. Han LP, Li CJ, Sun B, Xie Y, Guan Y, Ma ZJ and Chen LM: Protective effects of celastrol on diabetic liver injury via TLR4/MyD88/NF- $\mathrm{kB}$ signaling pathway in type 2 diabetic rats. J Diabetes Res 2016: 2641248, 2016.

17. Barberà-Cremades M,Baroja-Mazo A and Pelegrín P: Purinergic signaling during macrophage differentiation results in M2 alternative activated macrophages. J Leukoc Biol 99: 289-299, 2016.

18. Chávez-Sánchez L, Garza-Reyes MG, Espinosa-Luna JE, Chávez-Rueda K, Legorreta-Haquet MV and Blanco-Favela F: The role of TLR2, TLR4 and CD36 in macrophage activation and foam cell formation in response to oxLDL in humans. Hum Immunol 75: 322-329, 2014

19. Rittirsch D, Huber-Lang MS, Flierl MA and Ward PA: Immunodesign of experimental sepsis by cecal ligation and puncture. Nat Protoc 4: 31-36, 2009.

20. Zhang Z, Watt NJ, Hopkins J, Harkiss G and Woodall CJ: Quantitative analysis of maedi-visna virus DNA load in peripheral blood monocytes and alveolar macrophages. J Virol Methods 86: 13-20, 2000.

21. Thompson JH and Richter WR: Hemotoxylin-eosin staining adapted to automatic tissue processing. Stain Technol 35: 145-148, 1960.

22. Tuo YL, Li XM and Luo J: Long noncoding RNA UCA1 modulates breast cancer cell growth and apoptosis through decreasing tumor suppressive miR-143. Eur Rev Med Pharmacol Sci 19: 3403-3411, 2015

23. Wang Y, Chen G, Yu X, Li Y, Zhang L, He Z, Zhang N, Yang X, Zhao Y, Li N and Qiu H: Salvianolic acid B ameliorates cerebral ischemia/reperfusion injury through inhibiting TLR4/MyD88 signaling pathway. Inflammation 39: 1503-1513, 2016.

24. Huang C, Pan L, Lin F, Qian W and Li W: Alveolar macrophage TLR4/MyD88 signaling pathway contributes to ventilator-induced lung injury in rats. Xi Bao Yu Fen Zi Mian Yi Xue Za Zhi 31: 182-189, 2015 (In Chinese).

25. Loyau J, Malinge P, Daubeuf B, Shang L, Elson G, Kosco-Vilbois M, Fischer N and Rousseau F: Maximizing the potency of an anti-TLR4 monoclonal antibody by exploiting proximity to Fc $\gamma$ receptors. MAbs 6: 1621-1630, 2014.

26. Odegaard JI and Chawla A: Alternative macrophage activation and metabolism. Annu Rev Pathol 6: 275-297, 2011.

27. Gong D, Shi W, Yi SJ, Chen H, Groffen J and Heisterkamp N: TGF $\beta$ signaling plays a critical role in promoting alternative macrophage activation. BMC Immunol 13: 31, 2012. 
28. Jing X, Chen SS, Jing W, Tan Q, Yu MX and Tu JC: Diagnostic potential of differentially expressed Homer1, IL-1 $\beta$ and TNF- $\alpha$ in coronary artery disease. Int J Mol Sci 16: 535-546, 2014.

29. Lin X, Kong J, Wu Q, Yang Y and Ji P: Effect of TLR4/MyD88 signaling pathway on expression of IL- $1 \beta$ and TNF- $\alpha$ in synovial fibroblasts from temporomandibular joint exposed to lipopolysaccharide. Mediators Inflamm 2015: 329405, 2015

30. Ware LB, Koyama T, Zhao Z, Janz DR, Wickersham N, Bernard GR, May AK, Calfee CS and Matthay MA: Biomarkers of lung epithelial injury and inflammation distinguish severe sepsis patients with acute respiratory distress syndrome. Crit Care 17: R253, 2013.

31. Terpstra ML, Aman J, van Nieuw Amerongen GP and Groeneveld AB: Plasma biomarkers for acute respiratory distress syndrome: A systematic review and meta-analysis*. Crit Care Med 42: 691-700, 2014.

32. Escoubet-Lozach L, Benner C, Kaikkonen MU, Lozach J, Heinz S, Spann NJ, Crotti A, Stender J, Ghisletti S, Reichart D, et al: Mechanisms establishing TLR4-responsive activation states of inflammatory response genes. PLoS Genet 7: e1002401, 2011.
33. Dai H, Pan L, Lin F, Ge W, Li W and He S: Mechanical ventilation modulates Toll-like receptors 2,4 , and 9 on alveolar macrophages in a ventilator-induced lung injury model. J Thorac Dis 7: 616-624, 2015.

34. Chuffa LG, Fioruci-Fontanelli BA, Mendes LO, Ferreira Seiva FR, Martinez M, Fávaro WJ, Domeniconi RF, Pinheiro PF, Delazari Dos Santos L and Martinez FE: Melatonin attenuates the TLR4-mediated inflammatory response through MyD88and TRIF-dependent signaling pathways in an in vivo model of ovarian cancer. BMC Cancer 15: 34, 2015.

This work is licensed under a Creative Commons Attribution-NonCommercial-NoDerivatives 4.0 International (CC BY-NC-ND 4.0) License. 